2016 145th TMS Annual Meeting \& Exhibition, REWAS 2016 Symposium, February 14 - 18, 2016, Nashville, Tennessee

\title{
3D PRINTED ABS AND CARBON FIBER REINFORCED POLYMER SPECIMENS FOR ENGINEERING EDUCATION
}

\author{
Michael Golub ${ }^{1}$, Xingye Guo ${ }^{1}$, Mingyo Jung ${ }^{2}$, Jing Zhang ${ }^{1 *}$ \\ ${ }^{1}$ Department of Mechanical Engineering, Indiana University- Purdue University Indianapolis, \\ Indianapolis, IN 46202, USA \\ ${ }^{2}$ University High School, 2825 W. 116th Street, Carmel, IN 46032, USA \\ *Corresponding author: jz29@iupui.edu; 317-278-7186
}

Keywords: 3D Printing, Additive Manufacturing, ABS, ABS plus, Carbon Fiber Reinforced Polymer, Mechanical Property

\begin{abstract}
Three 3D printed plastic materials, ABS, ABS plus, and CFRP, have been studied for their potential applications in engineering education. Using tensile test, the stress strain curves of the materials have been measured. The Young's modulus, ultimate strength, and fracture toughness of the materials are calculated from the stress strain curve. The results show that CFRP has the highest stiffness or Young's modulus. ABS plus has strongest mechanical properties, with highest ultimate strength and fracture toughness. With the measured properties, the 3D printed samples are a viable solution for engineering students to learn mechanical properties of materials.
\end{abstract}

\section{Introduction}

Fused deposition modeling (FDM) is an additive manufacturing technology commonly used for modeling, prototyping, and production applications. It is one of the techniques used for 3D printing. FDM works on an "additive" principle by laying down material in layers; a plastic filament or metal wire is unwound from a coil and supplies material to produce a part [1]. 3D printers that run on FDM Technology build parts layer-by-layer from the bottom up by heating and extruding thermoplastic filament [2]. The process is simple: (1) Pre-processing: Buildpreparation software slices and positions a 3D CAD file and calculates a path to extrude thermoplastic and any necessary support material. (2) Production: The 3D printer heats the thermoplastic to a semi-liquid state and deposits it in ultra-fine beads along the extrusion path. Where support or buffering is needed, the $3 \mathrm{D}$ printer deposits a removable material that acts as 
scaffolding. (3) Post-processing: The user breaks away support material or dissolves it in detergent and water, and the part is ready to use [2].

In this study, the study is focused on $3 \mathrm{D}$ printed ABS, ABS plus, and carbon fiber reinforced polymer. Their mechanical properties, including Young's modulus, ultimate strength, and fracture toughness, are characterized and compared using tensile test.

\section{Methodology}

Three plastic materials are used in this study: ABS from Stratasys (Eden Prairie, MN), ABS plus from the 3D Filament Shop Ltd. (the Swan Centre, Higher Swan Lane, Bolton, UK), and carbon fiber reinforced polymer (CFRP, $20 \mathrm{wt} \%$ of carbon fiber) from 3DXTech (Wyoming, MI). At least three samples of each material are used, and only the average mechanical properties are reported below. The specimens used in this study are designed in accordance with the ASTM standard test method for tensile properties of plastics. The printer used is FDM 3D printer developed by 3D Parts Manufacturing LLC (Indianapolis, Indiana).

The tensile testing is done using a MTS Systems universal testing machine according to ASTM standards for tensile properties of plastics. The model number of extensometer used in the test is 634.12E-54.

Before the tensile tests, the width and thickness of the center section of each of the specimens are measured then entered into the testing program. The tensile specimen is loaded into the testing machine by attaching the clamps to both ends and the distance between the clamps is measured and entered into the program. The tensile strain rate applied is $0.2 \mathrm{in} / \mathrm{min}(0.0847 \mathrm{~mm} / \mathrm{s})$. The program records tensile load and elongation, which can be converted to stress - strain curves.

\section{Results and discussion}

The fractured tensile samples are shown in Figure 1. In general, the samples show brittle fracture due to relatively small deformation. In order to evaluate the mechanical properties, the stressstrain curves of the three samples are plotted in Figure 2. 


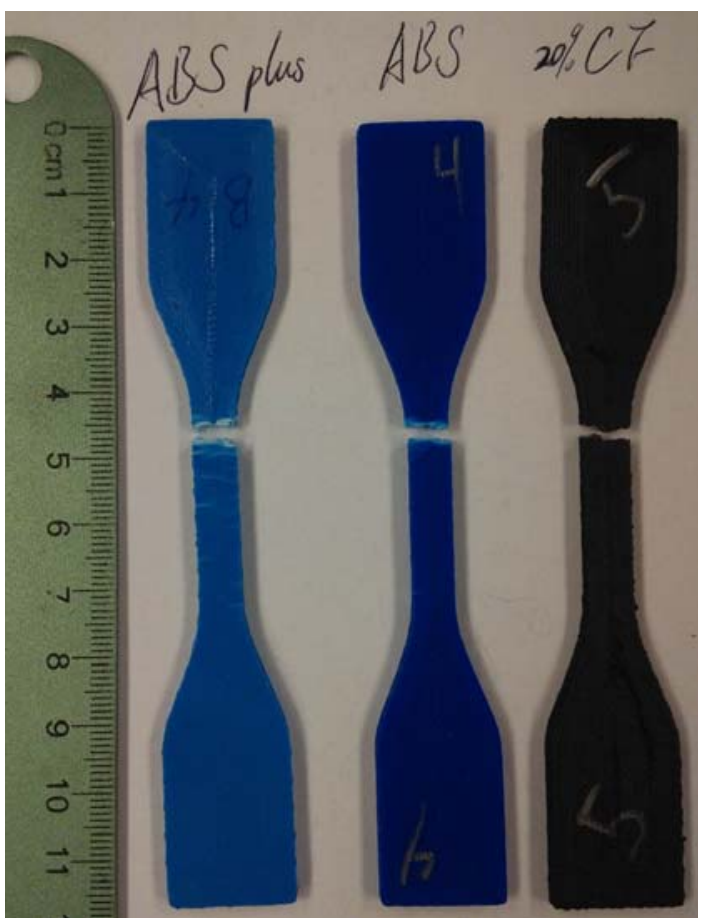

Figure 1: Optical images of the fracture tensile testing samples.

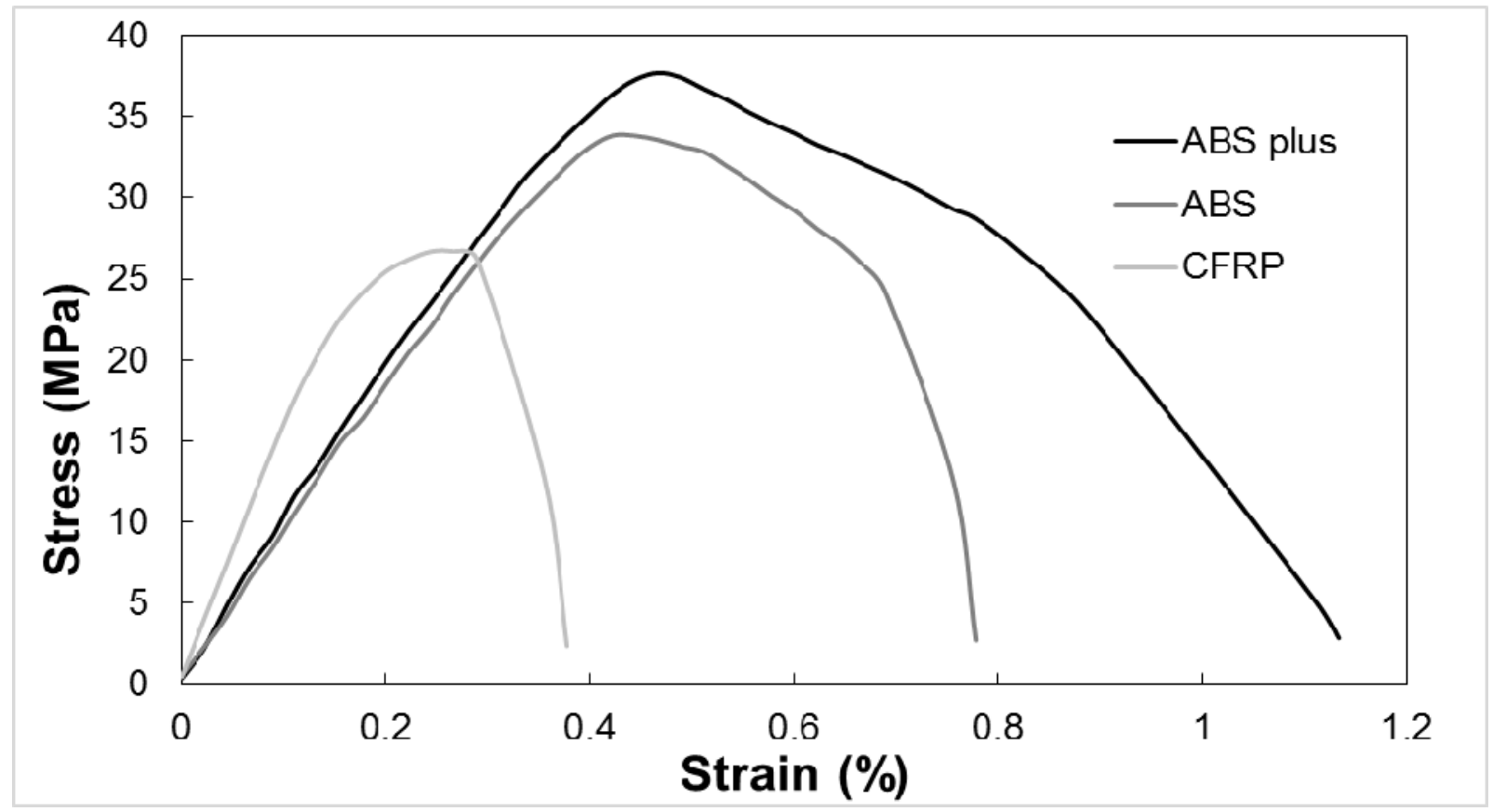

Figure 2: Representative stress-strain curves of tensile tests of three materials

The mechanical properties of the three materials, derived from the stress-strain curves in Figure 2, are summarized in Table 1. CFRP has the highest Young's modulus, due to its inclusion of high stiffness carbon fibers. However, its ultimate strength and fracture toughness are lower than those of ABS and ABS plus. So 3D printed CFRP is suitable for rigid applications. ABS and 
ABS plus have similar Young's modulus, but ABS plus has much higher ultimate strength and fracture toughness. Therefore, ABS plus is suitable for large deformation applications, but more expensive than ABS. The order of cost of the three materials is: ABS $<$ ABS plus $<$ CFRP.

Table 1: Averaged mechanical properties of 3D printed ABS, ABS plus, and CFRP

\begin{tabular}{|l|l|l|l|}
\hline & $\begin{array}{l}\text { Young's modulus } \\
(\mathrm{GPa})\end{array}$ & $\begin{array}{l}\text { Ultimate strength } \\
(\mathrm{MPa})\end{array}$ & $\begin{array}{l}\text { Fracture toughness } \\
(\mathrm{MPa})\end{array}$ \\
\hline ABS plus & 9.8 & 37.7 & 0.286 \\
\hline ABS & 9.2 & 33.9 & 0.179 \\
\hline CFRP & 17.1 & 26.7 & 0.073 \\
\hline
\end{tabular}

\section{Educational plan}

With the measured materials properties, the educational plan of using the 3D printed plastics in engineering curriculums would be the following. The low cost of the $3 \mathrm{D}$ printed plastic samples allows student groups can print multiple samples each. They can use the built-in software to do the standard deviation of sample dimensions and manually check it. With steel, brass or aluminum the costs are too high to get enough samples to do a standard deviation.

Also because student can have more samples, they will gain experience performing the tensile tests. Previously only student teaching assistant used the tensile testing machine and extensometer since only one sample was available. Now the students can perform the experiment themselves since more samples are available. It is performed under close supervision. This is done so not to harm the equipment and to make sure that the multiple samples are done accurately.

As we acquire the technology to make different plastic samples we can add them to the lab session. We have not found a replacement for the metals, and are still doing a few of the metals per year so the students have comparison data. It is important that students understand the small differences in plastic deformation and fracture but they need to see and experience a larger spectrum of materials. Therefore, 3D printed plastic samples are a viable solution for engineering students to learn mechanical properties of materials.

\section{Summary}

Three 3D printed plastic materials, ABS, ABS plus, and CFRP, are investigated in this study:

1. CFRP has the highest stiffness or Young's modulus

2. ABS plus has strongest mechanical properties, with highest ultimate strength and fracture toughness.

3. 3D printed samples are a viable solution for engineering students to learn mechanical properties of materials.

\section{Acknowledgements}


JZ acknowledges the financial support provided by Walmart Foundation (project title: Optimal Plastic Injection Molding Tooling Design and Production through Advanced Additive Manufacturing).

\section{References}

1. Wikipedia. http://en.wikipedia.org/wiki/.

2. Stratasys. Available from: http://usglobalimages.stratasys.com/Main/Files/ White\%20Papers/WP_FDM_3D\%20PrintingWithFDM.pdf?v=635786177472811876. 